TRANSACTIONS OF THE

AMERICAN MATHEMATICAL SOCIETY

Volume 351, Number 2, February 1999, Pages 515-531

S 0002-9947(99)01965-0

\title{
THE STABILITY OF THE EQUILIBRIUM OF REVERSIBLE SYSTEMS
}

\author{
BIN LIU
}

ABStract. In this paper, we consider the system

$$
\dot{x}=a(t) y^{2 m+1}+f_{1}(x, y, t), \quad \dot{y}=-b(t) x^{2 n+1}+f_{2}(x, y, t),
$$

where $m, n \in \mathbf{Z}_{+}, m+n \geq 1, a(t)$ and $b(t)$ are continuous, even and 1-periodic in the time variable $t ; f_{1}$ and $f_{2}$ are real analytic in a neighbourhood of the origin $(0,0)$ of $(x, y)$-plane and continuous 1 -periodic in $t$. We also assume that the above system is reversible with respect to the involution $G:(x, y) \mapsto$ $(-x, y)$. A sufficient and necessary condition for the stability in the Liapunov sense of the equilibrium $(x, y)=(0,0)$ is given.

\section{INTRODUCTION}

In this paper, we are concerned with the stability in the Liapunov sense of the equilibrium $(x, y)=(0,0)$ for the system

$$
\dot{x}=F_{1}(x, y, t), \quad \dot{y}=F_{2}(x, y, t)
$$

where $F_{1}$ and $F_{2}$ are continuous and 1-periodic functions in the time $t$ and $F_{1}(0,0, t)$ $=F_{2}(0,0, t)=0$. Since Liapunov's times, many authors have studied this problem.

The linearization of (1.1) around the origin is of the form

$$
\dot{x}=a_{11}(t) x+a_{12}(t) y, \quad \dot{y}=b_{11}(t) x+b_{12}(t) y .
$$

If the system (1.2) can be written into the form

$$
\dot{z}=J A(t) z,
$$

where $z=$ col. $(x, y)$ and $J=\left(\begin{array}{cc}0 & -1 \\ 1 & 0\end{array}\right)$ is the standard symplectic matrix, i.e., (1.2) is a linear Hamiltonian system. Then, it is well known that the stability of the equilibrium $(0,0)$ of $(1.2)$ is closely related to the position in the plane of the Floquet multipliers $\lambda_{1}, \lambda_{2}$. When $\lambda_{j} \in \mathbf{S}^{1}, \lambda_{j} \neq \pm 1$, i.e., in the elliptic case, the equilibrium is strongly stable in the sense of Krein; when $\lambda_{j} \notin \mathbf{S}^{1}$, that is, in the hyperbolic case, it is unstable. Some criteria for stability can be found in [1] and [4]. For example, when $A(t)=\left(\begin{array}{cc}0 & c(t) \\ -1 & 0\end{array}\right)$, that is, the system (1.2) is the classical Hill's equation, the equilibrium is stable under the following condition:

$$
\min _{t} c(t)>0, \quad \int_{0}^{1} c(t) d t<4 .
$$

Received by the editors March 17, 1995 and, in revised form, December 4, 1995.

1991 Mathematics Subject Classification. Primary 58F13.

Key words and phrases. Reversible systems, stability, invariant curves.

Research was supported by the NNSF of China.

(C)1999 American Mathematical Society 
In 1994, the stability of the equilibrium of the nonlinear equation

$$
\ddot{x}+c(t) x+a(t) x^{2 n+1}+\cdots=0,
$$

was studied by R. Ortega in [7]. Using the Moser twist theorem, he proved that the trivial solution $x=0$ is stable if

(i) the equilibrium of the equation (1.2) is stable;

(ii) $a(t)>0$ or $a(t)<0$.

Recently, in [3] we obtained a sufficient and necessary condition for the following equation:

$$
\ddot{x}+a(t) x^{2 n+1}+e(t, x)=0, \quad n \geq 1,
$$

where $a(t), e(t, x)$ are continuous and 1-periodic functions in $t, e(t, x)$ is smooth in $x$ and $|e(t, x)|=O\left(|x|^{2 n+2}\right)$ in a neighborhood of $x=0$. The result is:

If $\int_{0}^{1} a(t) d t \neq 0$, then the trivial solution $x=0$ of the equation (1.5) is stable if and only if $\int_{0}^{1} a(t) d t>0$.

Remark. In contrast to Ortega's result, in [3] we do not need the stability of the trivial solution of the corresponding linearization of the equation (1.5). In fact, the corresponding linearization equation of (1.5) is $\ddot{x}=0$. Obviously, its trivial solution $x=0$ is unstable.

In this paper, we are concerned with the stability in the Liapunov sense of the trivial solution $(x, y)=(0,0)$ of the differential equations

$$
\begin{aligned}
& \dot{x}=a(t) y^{2 m+1}+f_{1}(x, y, t), \\
& \dot{y}=-b(t) x^{2 n+1}+f_{2}(x, y, t),
\end{aligned}
$$

where $m, n \in \mathbf{Z}_{+}, m+n \geq 1, a(t)$ and $b(t)$ are continuous, even and 1-periodic functions in the time variable $t ; f_{1}$ and $f_{2}$ are real analytic in a neighbourhood of the origin $(0,0)$ of $(x, y)$-plane and continuous 1-periodic in $t$. We also assume that the above system is reversible with respect to the involution $G:(x, y) \mapsto(-x, y)$. The definition of reversible systems can be found in the appendix.

From the fact that the system of (1.6) is reversible with respect to $G$, it follows that

$$
\begin{aligned}
& f_{1}(-x, y,-t)=f_{1}(x, y, t), \\
& f_{2}(-x, y,-t)=-f_{2}(x, y, t) .
\end{aligned}
$$

Assume that $g(x, y, t)$ is a real analytic function in a neighbourhood of the origin $(0,0)$ of $(x, y)$-plane, that is, its Taylor series

$$
g(x, y, t)=\sum_{i \geq k, j \geq l} g_{i j}(t) x^{i} y^{j}, \quad k, l \in \mathbf{Z}_{+},
$$

converges in this neighbourhood.

Definition. Assume $g$ is a real analytic in a neighbourhood of the origin $(0,0)$ of $(x, y)$-plane and its Taylor series is given in (1.8). We say the function $g$ satisfies the property $\mathcal{P}(m, n)$ if the following inequality

$$
k(m+1)+l(n+1)>\max \{(2 n+1)(m+1),(2 m+1)(n+1)\}
$$

holds.

Remark. If $m=n=0$ and the function $g(x, y)$ satisfies the property $\mathcal{P}(0,0)$, then $g=O\left(x^{2}+y^{2}\right)$. 
In this paper, we will prove the following theorem.

Theorem 1. Assume that $\int_{0}^{1} a(t) d t \cdot \int_{0}^{1} b(t) d t \neq 0$ and $f_{1}, f_{2}$ satisfy the property $\mathcal{P}(m, n)$. Then the following statements are equivalent:

(1) The trivial solution $(x, y)=(0,0)$ of the equation (1.6) is stable;

(2) $\int_{0}^{1} a(t) d t \cdot \int_{0}^{1} b(t) d t>0$.

Moreover, if $\int_{0}^{1} a(t) d t \cdot \int_{0}^{1} b(t) d t>0$ there is at least two subharmonic solutions of (1.6) with minimal period $k$ for every sufficiently large $k \in \mathbf{N}$ in a neighborhood of the equilibrium of (1.6).

Remark. If $m=1, n=0$, then the linearized equation of (1.6) is of the form

$$
\dot{x}=0, \quad \dot{y}=-b(t) x .
$$

The equilibrium $(x, y)=(0,0)$ of this system is unstable. From our results, the trivial solution of the whole system (1.6) is stable if $\int_{0}^{1} a(t) d t \cdot \int_{0}^{1} b(t) d t>0$.

The rest of this paper is organized in the following way. In $\S 2$ we introduce the action-angle variables and prove the stability of the trivial solution $(x, y)=$ $(0,0)$ of the equation (1.6) when $\int_{0}^{1} a(t) d t \cdot \int_{0}^{1} b(t) d t>0$. In $\S 3$, we prove that if $\int_{0}^{1} a(t) d t \cdot \int_{0}^{1} b(t) d t<0$, then the trivial solution $(x, y)=(0,0)$ of $(1.6)$ is unstable. Some definitions and properties for reversible systems are listed in the appendix.

In what follows, to say that a solution of a differential equation or a fixed point of a homeomorphism is stable in the sense of Liapunov will mean that it is Liapunov stable in the future and also in the past. If it is not stable it will be called unstable.

\section{ACTION-ANGLE VARIABLES AND THE PROOF OF THE STABILITY}

In this section, based on the theory of stability of fixed points of mappings in the plane, we prove that, under the condition

$$
\int_{0}^{1} a(t) d t \cdot \int_{0}^{1} b(t) d t>0,
$$

in every small neighborhood of the trivial solution $(x(t), y(t))=(0,0)$, there are invariant curves of Poincaré mapping of (1.6). These curves are around the origin $(0,0)$ in the $(x, y)$-plane which yield the stability of the trivial solution $(x, y)=$ $(0,0)$.

Without loss of generality, we assume that

$$
\int_{0}^{1} a(t) d t>0, \quad \int_{0}^{1} b(t) d t>0 .
$$

Let $c_{1}=\int_{0}^{1} a(t) d t>0, c_{2}=\int_{0}^{1} b(t) d t>0$. Then

$$
a(t)=c_{1}+a_{1}(t), \quad b(t)=c_{2}+b_{1}(t)
$$

and

$$
\int_{0}^{1} a_{1}(t) d t=0, \quad \int_{0}^{1} b_{1}(t) d t=0 .
$$

Moreover, $a_{1}(t)$ and $b_{1}(t)$ are even in $t$.

Furthermore, we assume that $c_{1}=c_{2}=1$. Hence,

$$
a(t)=1+a_{1}(t), \quad b(t)=1+b_{1}(t) .
$$


Remark. If $c_{1}<0$ and $c_{2}<0$, then, let $\tau=-t$, we obtain that

$$
\begin{aligned}
& \frac{d x}{d \tau}=\hat{a}(\tau) y^{2 m+1}+g_{1}(x, y, \tau), \\
& \frac{d y}{d \tau}=-\hat{b}(\tau) x^{2 n+1}+g_{2}(x, y, \tau),
\end{aligned}
$$

where $(\hat{a}(\tau), \hat{b}(\tau))=-(a(-t), b(-t))$. This system is also reversible with respect to $G:(x, y) \mapsto(-x, y)$ and

$$
\int_{0}^{1} \hat{a}(\tau) d \tau>0, \quad \int_{0}^{1} \hat{b}(\tau) d \tau>0
$$

If $\left(c_{1}, c_{2}\right) \neq(1,1)$, we can introduce the variables $(u, v)$ by

$$
u=\gamma_{1} x, \quad v=\gamma_{2} y .
$$

Choose the positive constants $\gamma_{1}, \gamma_{2}$ such that

$$
\frac{\gamma_{2}^{2 m+1}}{\gamma_{1}} \cdot c_{1}=\frac{\gamma_{1}^{2 n+1}}{\gamma_{2}} \cdot c_{2}=1
$$

Under this transformation, the system (1.6) is transformed into the form

$$
\dot{u}=c(t) v^{2 m+1}+p(u, v, t), \quad \dot{v}=-d(t) u^{2 n+1}+q(u, v, t) .
$$

where $\int_{0}^{1} c(t) d t=\int_{0}^{1} d(t) d t=1$

Now, we consider the auxiliary system

$$
\dot{x}=y^{2 m+1}, \quad \dot{y}=-x^{2 n+1} .
$$

The system (2.1) is a Hamiltonian system

$$
\dot{x}=\frac{\partial h}{\partial y}, \quad \dot{y}=-\frac{\partial h}{\partial x},
$$

with the Hamiltonian

$$
h(x, y)=\frac{1}{2 m+2} \cdot y^{2 m+2}+\frac{1}{2 n+2} \cdot x^{2 n+2} .
$$

Clearly, $h>0$ on $\mathbf{R}^{2}$ except at the only equilibrium $(x, y)=(0,0)$ where $h=0$.

Let $(S(t), C(t))$ be the solution of the system (2.1) satisfying the initial condition: $(S(0), C(0))=(0,1)$. This solution is a periodic solution. Let $T_{0}$ be its minimal period. We have

$$
0<T_{0}=4 \int_{0}^{1} \frac{1}{\left[\frac{n+1}{m+1}\left(1-s^{2 m+2}\right)\right]^{\frac{2 n+1}{2 n+2}}} d s<+\infty
$$

and the analytic functions $S(t)$ and $C(t)$ satisfy

(i) $(S(-t), C(-t))=(-S(t), C(t))$ and $\left(S\left(t+T_{0}\right), C\left(t+T_{0}\right)\right)=(S(t), C(t))$.

(ii) $\frac{1}{2 n+2} S^{2 n+2}(t)+\frac{1}{2 m+2} C^{2 m+2}(t)=\frac{1}{2 m+2}, \forall t \in \mathbf{R}$.

(iii) $\frac{d}{d t} S=C^{2 m+1}, \frac{d}{d t} C=-S^{2 n+1}$.

Suppose that $(u(t), v(t))$ is a solution of $(2.1)$ with the initial condition:

$$
(u(0), v(0))=(0, \gamma) \text {. }
$$

Then, we have

$$
\begin{aligned}
& u(t)=\gamma^{\frac{m+1}{n+1}} S\left(\gamma^{\kappa} t\right), \\
& v(t)=\gamma C\left(\gamma^{\kappa} t\right),
\end{aligned}
$$


where $\kappa=\frac{2 m n+m+n}{n+1}>0$. Hence, all the solutions of (2.1) are periodic, and the periods tending to zero as $h=h_{0}=\frac{1}{2 m+2} \cdot \gamma^{2 m+2}$ tends to infinity.

In the following, we introduce an action-angle variable $(\rho, \theta)$. Define a transformation $\Phi: \mathbf{R}^{+} \times \mathbf{S}^{1} \rightarrow \mathbf{R}^{2},(\rho, \theta) \mapsto(x, y)$ :

$$
\Phi: x=d_{1}^{\alpha} \rho^{\alpha} S\left(\theta T_{0}\right), \quad y=d_{1}^{\beta} \rho^{\beta} C\left(\theta T_{0}\right),
$$

where $\alpha=\frac{m+1}{m+n+2}, \beta=1-\alpha=\frac{n+1}{m+n+2}$ and $d_{1}=\frac{m+n+2}{(n+1) T_{0}}$. Hence, we have $(n+1) \alpha=(m+1) \beta$.

One can easily verify that the transformation $\Phi$ is a symplectic mapping. In fact,

$$
\begin{aligned}
\left|\frac{\partial(x, y)}{\partial(\rho, \theta)}\right| & =\left|\alpha T_{0} d_{1}^{\alpha+\beta} \rho^{\alpha+\beta-1} \cdot S^{2 n+2}\left(\theta T_{0}\right)+\beta T_{0} d_{1}^{\alpha+\beta} \rho^{\alpha+\beta-1} C^{2 m+2}\left(\theta T_{0}\right)\right| \\
& =\frac{1}{m+n+2} \cdot\left|T_{0} d_{1} \cdot\left((n+1) C^{2 m+2}\left(\theta T_{0}\right)+(m+1) S^{2 n+2}\left(\theta T_{0}\right)\right)\right| \\
& =1 .
\end{aligned}
$$

Under this transformation, the system (2.1) is transformed into the simpler form

$$
\dot{\rho}=-\frac{\partial h_{0}}{\partial \theta}=0, \quad \dot{\theta}=\frac{\partial h_{0}}{\partial \rho}=c \cdot \rho^{2(m+1) \beta-1},
$$

where $h_{0}(\rho, \theta, t)=\frac{c}{(2 m+2) \beta} \cdot \rho^{2(m+1) \beta}$ and $c=d_{1}^{2(m+1) \beta} \cdot \beta$.

The system (1.6) is transformed into the form

$$
\begin{aligned}
& \dot{\rho}=h_{1}(\rho, \theta, t)+h_{2}(\rho, \theta, t), \\
& \dot{\theta}=c \cdot \rho^{(2 m+2) \beta-1}+g_{1}(\rho, \theta, t)+g_{2}(\rho, \theta, t),
\end{aligned}
$$

where

$$
\begin{aligned}
& h_{1}(\rho, \theta, t)=T_{0} d_{1}^{(2 m+2) \beta} \rho^{(2 m+2) \beta}\left[a(t) S^{2 n+1} C^{2 m+1}-b(t) S^{2 n+1} C^{2 m+1}\right], \\
& h_{2}(\rho, \theta, t)=T_{0} d_{1}^{\beta} \rho^{\beta} S^{2 n+1} f_{1}+T_{0} d_{1}^{\alpha} \rho^{\alpha} C^{2 m+1} f_{2}, \\
& g_{1}(\rho, \theta, t)=d_{1}^{(2 m+2) \beta} \rho^{(2 m+2) \beta-1}\left(\alpha a_{1}(t) S^{2 n+2}\left(\theta T_{0}\right)+\beta b_{1}(t) C^{2 m+2}\left(\theta T_{0}\right)\right), \\
& g_{2}(\rho, \theta, t)=-\alpha d_{1}^{\alpha} \rho^{\alpha-1} S\left(\theta T_{0}\right) f_{2}+\beta d_{1}^{\beta} \rho^{\beta-1} C\left(\theta T_{0}\right) f_{1} .
\end{aligned}
$$

with $f_{i}=f_{i}\left(d_{1}^{\alpha} \rho^{\alpha} S\left(\theta T_{0}\right), d_{1}^{\beta} \rho^{\beta} C\left(\theta T_{0}\right), t\right), i=1,2$.

From the equality (1.7) and the properties of the functions $S$ and $C$, it follows that

$$
\begin{aligned}
& h_{1}(\rho,-\theta,-t)=-h_{1}(\rho, \theta, t), \\
& h_{2}(\rho,-\theta,-t)=-h_{2}(\rho, \theta, t), \\
& g_{1}(\rho,-\theta,-t)=g_{1}(\rho, \theta, t), \\
& g_{2}(\rho,-\theta,-t)=g_{2}(\rho, \theta, t) .
\end{aligned}
$$

Hence, the transformed system (2.5) is also reversible with respect to the involution $\mathcal{G}:(\rho, \theta) \mapsto(\rho,-\theta)$.

Because $\int_{0}^{1} a_{1}(t) d t=\int_{0}^{1} b_{1}(t) d t=0$, we have

$$
\int_{0}^{1} g_{1}(\rho, \theta, t) d t=0 .
$$

We introduce a space of functions, denoted by $\mathcal{F}(r)$, which behave for $0<\lambda \leq \lambda_{0}$, with all their derivatives, like $\lambda^{r} p(\theta)$. 
Definition 2.1. For a given constant $r \in \mathbf{R}, f(\lambda, \theta) \in \mathcal{F}(r)$ if $f \in C^{\infty}\left(R^{+} \times S^{1}\right)$ and, for all nonnegative integers $j$ and $l$, we have

$$
\sup _{0<\lambda \leq \lambda_{j, l}, \theta \in S^{1}}\left(\lambda^{j-r}\left|\left(D_{\lambda}^{j}\right)\left(D_{\theta}^{l}\right) f(\lambda, \theta)\right|\right)<+\infty,
$$

where $\lambda_{j, l}$ is some positive constant.

We give some basic properties of the space $\mathcal{F}(r)$.

Lemma 2.1. The following conclusions hold:

(i) if $r_{1}<r_{2}$, then $\mathcal{F}\left(r_{2}\right) \subset \mathcal{F}\left(r_{1}\right)$;

(ii) if $f \in \mathcal{F}(r)$, then $\left(D_{\lambda}^{j}\right) f \in \mathcal{F}(r-j)$;

(iii) if $f_{1} \in \mathcal{F}\left(r_{1}\right)$ and $f_{2} \in \mathcal{F}\left(r_{2}\right)$, then $f_{1} \cdot f_{2} \in \mathcal{F}\left(r_{1}+r_{2}\right)$;

(iv) if $f \in \mathcal{F}(r)$ satisfying $|f(\lambda, \cdot)| \geq c_{0} \lambda^{r}$ for $0<\lambda \leq \lambda_{0}$, then $\frac{1}{r} f \in \mathcal{F}(-r)$.

Remark. The space $\mathcal{F}(r)$ defined here is similar to the space $\mathcal{F}(r)$ introduced by R. Dieckerhoff and E. Zehnder in [2].

Proof. Proofs of (i), (ii) and (iii) are immediate from the definition.

We now show (iv). Because $f \in \mathcal{F}(r)$, so $f \in C^{\infty}\left(\mathbf{R}^{+} \times \mathbf{S}^{1}\right)$. Let $g=\frac{1}{f}$. Then $\lambda^{r} \cdot|g| \leq \frac{1}{c_{0}}$.

Inductively, assuming that the estimates

$$
\sup _{\lambda \leq \lambda_{0}, \theta \in \mathbf{S}^{1}} \lambda^{j+r}\left|D_{\lambda}^{j} g\right|<\infty, \quad \text { and } j \leq n-1
$$

hold, we will prove the estimate for $j=n \geq 1$.

Since $f \cdot g=1$, then $D_{\lambda}^{n}(f \cdot g)=0$ for $n \geq 1$; that is,

$$
f \cdot D_{\lambda}^{n} g+\sum_{i+j=n, i \geq 1} \bar{c}_{i} D_{\lambda}^{i} f \cdot D_{\lambda}^{j} g=0,
$$

where $\bar{c}_{i}$ are positive constants, $i$ and $j$ are nonnegative integers. It follows that

$$
|f| \cdot\left|D_{\lambda}^{n} g\right| \leq \bar{C} \sum_{i+j=n, i \geq 1}\left|D_{\lambda}^{i} f\right| \cdot\left|D_{\lambda}^{j} g\right| .
$$

Then

$$
\lambda^{r} \cdot\left|D_{\lambda}^{n} g\right| \leq \frac{\bar{C}}{c_{0}} \sum_{i+j=n, i \geq 1}\left|D_{\lambda}^{i} f\right| \cdot\left|D_{\lambda}^{j} g\right| .
$$

Hence, we have

$$
\lambda^{n+r} \cdot\left|D_{\lambda}^{n} g\right| \leq \frac{\bar{C}}{c_{0}} \sum_{i+j=n, i \geq 1} \lambda^{i-r}\left|D_{\lambda}^{i} f\right| \cdot \lambda^{j+r}\left|D_{\lambda}^{j} g\right|,
$$

which together with the estimates $\lambda^{j+r} \cdot\left|D_{\lambda}^{j} g\right|<\infty$ (for $j \leq n-1$ ) yields

$$
\lambda^{n+r} \cdot\left|D_{\lambda}^{n} g\right|<\infty
$$

i.e.,

$$
\sup _{\lambda \leq \lambda_{0}, \theta \in \mathbf{S}^{1}} \lambda^{n+r}\left|D_{\lambda}^{n} g\right|<\infty
$$

Hence, $g \in \mathcal{F}(-r)$. 
From the equalities in (2.6) and the assumptions of our theorem, it follows that

$$
h_{1}(\rho, \theta, t) \in \mathcal{F}((2 m+2) \beta), g_{1}(\rho, \theta, t) \in \mathcal{F}((2 m+2) \beta-1),
$$

and $h_{2}=o\left(\rho^{(2 m+2) \beta}\right), g_{2}=o\left(\rho^{(2 m+2) \beta-1}\right)$.

Now, we define a transformation $\Psi:(\rho, \theta) \mapsto(\lambda, \phi)$ as follows

$$
\lambda=\rho, \quad \phi=\theta+f(\rho, \theta, t),
$$

where the function $f(\rho, \theta, t)$ will be determined later. Under this transformation, the system (2.5) is transformed into another reversible system with respect to $\mathcal{G}_{1}:(\lambda, \phi) \mapsto(\lambda,-\phi)$.

$$
\begin{aligned}
& \dot{\lambda}=\hat{h}_{1}(\lambda, \phi, t), \\
& \dot{\phi}=c \cdot \lambda^{(2 m+2) \beta-1}+\hat{g}_{1}(\lambda, \phi, t),
\end{aligned}
$$

where

$$
\begin{aligned}
\hat{h}_{1}(\lambda, \phi, t)= & h_{1}(\rho, \theta, t)+h_{2}(\rho, \theta, t), \\
\hat{g}_{1}(\lambda, \phi, t)= & g_{1}(\rho, \theta, t)+g_{2}(\rho, \theta, t)+\frac{\partial f}{\partial t} \\
& \quad+\frac{\partial f}{\partial \rho} \cdot\left(h_{1}+h_{2}\right)+\frac{\partial f}{\partial \theta} \cdot\left(d \cdot \rho^{(2 m+2) / \beta-1}+g_{1}+g_{2}\right) .
\end{aligned}
$$

Because $\int_{0}^{1} g_{1}(\rho, \theta, t) d t=0$, we can define the function $f$ :

$$
f(\rho, \theta, t)=-\int_{0}^{t} g_{1}(\rho, \theta, s) d s \in \mathcal{F}((2 m+2) \beta-1) .
$$

From (2.7), we have

$$
f(\rho,-\theta,-t)=-f(\rho, \theta, t) .
$$

Because $(2 m+2) \beta-1=\frac{2 m n+m+n}{m+n+2} \geq \frac{1}{3}$, the transformation $\Psi$ has an inverse $\Phi:(\lambda, \phi) \mapsto(\lambda,-\phi)$. $\Phi$ exists if $\lambda>0$ small. Moreover, the transformation $\Phi$ can be written into the form

$$
\Phi: \rho=\lambda, \quad \theta=\phi+r(\lambda, \phi, t)
$$

where $r=O\left(|\lambda|^{(2 m+2) \beta-1}\right)$. This transformation is a diffeomorphism on the cylinder

$$
A_{\lambda_{0}}=\left\{(\lambda, \phi) \mid \lambda \geq \lambda_{0}>0, \lambda \text { small, } \phi \in \mathbf{S}^{1}\right\},
$$

and a homeomorphism on $\left\{(\lambda, \phi) \mid \lambda \geq 0, \lambda\right.$ small, $\left.\phi \in \mathbf{S}^{1}\right\}$.

Now, we prove that

$$
r(\lambda,-\phi,-t)=-r(\lambda, \phi, t) .
$$

In fact, from the equality $\Phi \circ \Psi=\Psi \circ \Phi=\mathrm{id}$, it follows that

$$
r(\lambda, \phi, t)+f(\lambda, \phi+r(\lambda, \phi, t), t)=0 .
$$

Hence,

$$
\begin{aligned}
|r(\lambda,-\phi,-t)+r(\lambda, \phi, t)| & =|f(\lambda,-\phi+r(\lambda,-\phi,-t),-t)+f(\lambda, \phi+r(\lambda, \phi, t), t)| \\
& =|f(\lambda, \phi-r(\lambda,-\phi,-t), t)-f(\lambda, \phi+r(\lambda, \phi, t), t)| \\
& \leq \sup \left|\frac{\partial f}{\partial \theta}\right| \cdot \mid(r(\lambda,-\phi,-t)+r(\lambda, \phi, t) \mid .
\end{aligned}
$$


From $f \in \mathcal{F}((2 m+2) \beta-1)$, it follows that

$$
r(\lambda,-\phi,-t)=-r(\lambda, \phi, t),
$$

provided that $\lambda$ is sufficiently small.

By Lemma A.1, the system (2.11) is reversible with respect to $\mathcal{G}_{1}$.

Let $\xi=c \cdot \lambda^{(2 m+2) \beta-1}, \eta=\phi$. Then, (2.11) is transformed into

$$
\dot{\xi}=H_{1}(\xi, \eta, t), \quad \dot{\eta}=\xi+H_{2}(\xi, \eta, t),
$$

where $H_{1}(\xi, \eta, t)=o\left(\xi^{2}\right)$ and $H_{2}(\xi, \eta, t)=o(\xi)$. This system is also reversible to $\mathcal{G}_{2}:(\xi, \eta) \mapsto(\xi,-\eta)$.

One verifies easily that the solutions of (2.13) do exist for $0 \leq t \leq 2$ if the initial value $\lambda(0)=\lambda$ is sufficiently small.

We are now in a position to prove the stability of the equilibrium $(x, y)=(0,0)$ of (1.6).

Proposition 2.1. Suppose that $\int_{0}^{1} a(t) d t \cdot \int_{0}^{1} b(t) d t>0$. Then, the trivial solution $(x, y)=(0,0)$ of $(1.6)$ is stable in the sense of Liapunov. Moreover, there is at least two subharmonic solutions of (1.6) with minimal period $k$ for every sufficiently large $k \in N$ in a neighborhood of the equilibrium of (1.6).

In order to prove this proposition. The following celebrated Moser's twist theorem for reversible mappings is to be used.

Lemma 2.2 (Invariant curves of reversible mappings). Consider a mapping

$$
M_{\varepsilon}: \begin{aligned}
& r_{1}=r+\varepsilon^{\sigma} f(r, \theta, \varepsilon), \\
& \theta_{1}=\theta+\varepsilon^{\rho} \gamma(r)+\varepsilon^{\sigma} g(r, \theta, \varepsilon),
\end{aligned}
$$

where $0 \leq \rho<\sigma, 0 \leq \theta \leq 1,1 \leq r \leq 2$. Suppose that $M_{\varepsilon}$ is reversible with respect to $\Lambda:(r, \theta) \mapsto(r,-\theta)$ and $\gamma^{\prime}(r) \neq 0$. The functions $f$ and $g$ are assumed to be sufficiently often differentiable and 1-periodic in $\theta$. Then, for sufficiently small $\varepsilon$ there exists an invariant curve $\Gamma$ surrounding $r=1$. More precisely, given any number $\omega \in\left(\alpha+\varepsilon^{\rho} \gamma(1), \alpha+\varepsilon^{\rho} \gamma(2)\right)$ satisfying the inequalities

$$
\left|\omega-\frac{p}{q}\right| \geq c \varepsilon^{\rho}|q|^{-\frac{5}{2}}
$$

for all integers $p$ and $q \neq 0$, there exists a differentiable closed curve $\Gamma$

$$
r=F(\phi, \varepsilon), \quad \theta=\phi+G(\phi, \varepsilon)
$$

with $F$ and $G$ of period 1 in $\phi$, which is invariant under the mapping $M_{\varepsilon}$ and $\Lambda$ provided $\varepsilon$ is sufficiently small. The image point of a point on $\Gamma$ is obtained by replacing $\phi$ by $\phi+\omega$.

The proof of this lemma can be found in [6] and [8].

Proof of Proposition 2.1. First, we show that the trivial solution $(x, y)=(0,0)$ of (1.6) is stable.

From a classical result in the theory of fixed point of mappings in the plane, in order to prove the trivial solution $(x, y)=(0,0)$ is stable, it is enough to prove that there are invariant curves of the Poincaré mapping of the equation (1.6) in every small neighborhood of the solution $(x, y)=(0,0)$. This is equivalent to prove that there exist invariant curves of the Poincaré mapping of $(2.11)$ for $\xi \ll 1$. 
From the equation (2.11), one verifies easily that the solutions do exist for $0 \leq$ $t \leq 2$, if the initial value $\lambda(0)=\lambda$ is sufficiently small. Moreover, we can prove that the Poincaré mapping $\Pi$ of (2.11) is of the form

$$
\Pi: \begin{aligned}
& \lambda_{1}=\lambda+o\left(|\lambda|^{(2 m+2) \beta}\right), \\
& \phi_{1}=\phi+c \cdot \lambda^{(2 m+2) \beta-1}+o\left(|\lambda|^{(2 m+2) \beta}\right) .
\end{aligned}
$$

Replacing $\lambda$ by $\varepsilon r(1 \leq r \leq 2)$, the Poincaré mapping $\Pi$ is replaced by

$$
M: \begin{aligned}
& r_{1}=r+o\left(\varepsilon^{(2 m+2) \beta-1}\right), \\
& \phi_{1}=\phi+\varepsilon^{(2 m+2) \beta-1} \cdot c \cdot r^{(2 m+2) \beta-1}+o\left(\varepsilon^{(2 m+2) \beta-1}\right) .
\end{aligned}
$$

Because the equation (1.6) is reversible and the transformations $\Phi$ and $\Psi$ are invariant, the Poincaré mapping $\Pi$ is reversible. From Lemma 2.2, it follows that there is an invariant curve $\Gamma_{\varepsilon}$, under the mapping $M$, surrounding $r=1$ if $\varepsilon$ is sufficiently small. This means that the Poincaré mapping $\Pi$ of the system (2.11) has an invariant curve $\widetilde{\Gamma}_{\varepsilon}$ surrounding $\lambda=\varepsilon$ and in the annulus $\varepsilon<\lambda<2 \varepsilon$, which implies the stability of the trivial solution $(x, y)=(0,0)$ of the equation (1.6).

We next prove the existence of subharmonic solutions.

Consider the annulus $A$ bounded by two invariant curves of $\Pi$ with rotation numbers $\omega_{1}<\omega_{2}$. We map this annulus $A$ onto another annulus

$$
A_{0}=\{(\xi, \eta) \mid 0 \leq \eta \leq 1, \xi \bmod 1\} .
$$

If $\Psi_{j}$ are the embeddings of the invariant curves having rotation numbers $\omega_{j}$, we define the map $\chi: A_{0} \rightarrow A$ by

$$
\chi:(r, \theta) \mapsto\left(\eta \psi_{1}(\xi)+(1-\eta) \psi_{2}(\xi), \xi\right) .
$$

From Lemma 2.2, the embeddings $\psi_{j}$ are differentiably close to the injections of the circles $\omega_{j}=$ const., it follows that $\chi$ is a diffeomorphism. The induced map $J:=\chi^{-1} \circ \Pi \circ \chi$, expressed by

$$
J: \xi_{1}=f(\xi, \eta), \quad \eta_{1}=g(\xi, \eta),
$$

satisfies $f(\xi+1, \eta)=f(\xi, \eta)$ and $g(\xi+1, \eta)=g(\xi, \eta)$. It leaves the boundaries $\eta=0$ and $\eta=1$ invariant and

$$
f(\xi, 0)=\xi+\omega_{2}, \quad f(\xi, 1)=\xi+\omega_{1} .
$$

Define the map $\tau: A_{0} \rightarrow A_{0}$ by $\tau(\xi, \eta)=(\xi+1, \eta)$. To find periodic points of minimal period $q$ of $J$, for sufficiently large $q$, we let $q_{0}=\frac{1}{\left(\omega_{2}-\omega_{1}\right)^{2}}$. For $q \geq q_{0}$, choose an integer $p \in\left(q \omega_{1}, q \omega_{2}\right)$ such that $p$ and $q$ are relatively prime. The mapping $J_{q}:=\tau^{-p} \circ J^{q}$ on $A_{0}$, expressed by

$$
J_{q}: \xi_{1}=f_{q}(\xi, \eta), \quad \eta_{1}=g_{q}(\xi, \eta)
$$

satisfies

$$
f_{q}(\xi, 1)-\xi=-p+q \omega_{1}<0<-p+q \omega_{2}=f_{q}(\xi, 0)-\xi .
$$

From Theorem 5.1 in [8], we conclude that $J_{q}$ possesses at least two fixed points in $A_{0}$. Every such point corresponds to a periodic point of $J$, hence of $\Pi$, with minimal period $q$. The proof of this proposition is complete. 


\section{The Instability of THE EQUilibRium of (1.6)}

In this section, we prove that if $\int_{0}^{1} a(t) d t \int_{0}^{1} b(t) d t<0$, then the trivial solution $(x, y)=(0,0)$ of $(1.6)$ is unstable. Similar to the discussions in the above section, without loss of generality, we assume that

$$
a(t)=1+a_{1}(t), \quad b(t)=-1+b_{1}(t),
$$

where $\int_{0}^{1} a_{1}(t) d t=\int_{0}^{1} b_{1}(t) d t=0$. The equation (1.6) can be written into the following reversible system:

$$
\begin{aligned}
& \dot{x}=y^{2 m+1}+a_{1}(t) y^{2 m+1}+f_{1}(x, y, t), \\
& \dot{y}=x^{2 n+1}-b_{1}(t) x^{2 n+1}+f_{2}(x, y, t),
\end{aligned}
$$

where $m, n \in \mathbf{Z}_{+}, m+n \geq 1$.

We shall prove the following proposition.

Proposition 3.1. Suppose that

$$
a(t)=1+a_{1}(t), \quad b(t)=-1+b_{1}(t),
$$

where $\int_{0}^{1} a_{1}(t) d t=\int_{0}^{1} b_{1}(t) d t=0$ and $a_{1}(t), b_{1}(t)$ are even in $t$. Then the equilibrium point $(0,0)$ of $(1.6)$ is unstable.

In order to prove this proposition, we first define a transformation $\Phi: \mathbf{R}^{+} \times \mathbf{S}^{1} \rightarrow$ $\mathbf{R}^{2},(\rho, \theta) \mapsto(x, y):$

$$
\Phi: x=d_{1}^{\alpha} \rho^{\alpha} S\left(\theta T_{0}\right), \quad y=d_{1}^{\beta} \rho^{\beta} C\left(\theta T_{0}\right),
$$

where the constants $\alpha, \beta, d_{1}, T_{0}$ and functions $S, C$ are defined in $\S 2$.

Under the transformation $\Phi$, the system (3.1) is transformed into the form

$$
\begin{aligned}
& \dot{\rho}=c_{1} \rho^{(2 m+2) \beta} S^{2 n+1}\left(\theta T_{0}\right) C^{2 m+1}\left(\theta T_{0}\right)+\rho^{(2 m+2) \beta} h_{1}(\theta, t)+h_{2}(\rho, \theta, t), \\
& \dot{\theta}=c_{2} \rho^{(2 m+2) \beta-1} g(\theta)+\rho^{(2 m+2) \beta-1} g_{1}(\theta, t)+g_{2}(\rho, \theta, t),
\end{aligned}
$$

where

$$
\begin{aligned}
& h_{1}(\theta, t)=c_{3} S^{2 n+1}\left(\theta T_{0}\right) C^{2 m+1}\left(\theta T_{0}\right)\left(a_{1}(t)-b_{1}(t)\right), \\
& h_{2}(\rho, \theta, t)=c_{4} \rho^{\beta} S^{2 n+1}\left(\theta T_{0}\right) f_{1}+c_{5} \rho^{\alpha} C^{2 m+1}\left(\theta T_{0}\right) f_{2}, \\
& g(\theta)=\frac{n+1}{2(m+1)}-S^{2 n+2}\left(\theta T_{0}\right), \\
& g_{1}(\theta, t)=c_{6} S^{2 n+2}\left(\theta T_{0}\right) b_{1}(t)+c_{7} C^{2 m+2}\left(\theta T_{0}\right) a_{1}(t), \\
& g_{2}(\rho, \theta, t)=c_{8} \rho^{\beta-1} C\left(\theta T_{0}\right) f_{1}+c_{9} \rho^{\alpha-1} S\left(\theta T_{0}\right) f_{2},
\end{aligned}
$$

with $f_{i}=f_{i}\left(d_{1}^{\alpha} \rho^{\alpha} S\left(\theta T_{0}\right), d_{1}^{\beta} \rho^{\beta} C\left(\theta T_{0}\right), t\right), i=1,2 ; c_{1}, c_{2}, \ldots, c_{9}$ are constants and $c_{1}, c_{2}>0$.

The system (3.3) is reversible with respect to $G:(\rho, \theta) \mapsto(\rho,-\theta)$.

Lemma 3.1. There is a transformation $\Psi_{1}:(\rho, \theta) \mapsto(\rho, \phi)$. Under this transformation, the system (3.3) is transformed into the form

$$
\begin{aligned}
& \dot{\rho}=c_{1} \rho^{(2 m+2) \beta} S^{2 n+1}\left(\phi T_{0}\right) C^{2 m+1}\left(\phi T_{0}\right)+\rho^{(2 m+2) \beta} \xi_{1}(\phi, t)+\xi_{2}(\rho, \phi, t), \\
& \dot{\theta}=c_{2} \rho^{(2 m+2) \beta-1} g(\phi)+\eta_{1}(\rho, \phi, t),
\end{aligned}
$$


where

$$
\begin{aligned}
\xi_{1}(\phi, t)= & h_{1}(\phi, t) \\
\xi_{2}(\rho, \phi, t)= & c_{1} \rho^{(2 m+2) \beta}\left[S^{2 n+1}\left(\theta T_{0}\right) C^{2 m+1}\left(\theta T_{0}\right)-S^{2 n+1}\left(\phi T_{0}\right) C^{2 m+1}\left(\phi T_{0}\right)\right] \\
& \quad+\rho^{(2 m+2) \beta}\left[h_{1}(\theta, t)-h_{1}(\phi, t)\right]+h_{2}(\rho, \theta, t), \\
\eta_{1}(\rho, \phi, t)= & c_{2} \rho^{(2 m+2) \beta-1}[g(\theta)-g(\phi)]+g_{2}(\rho, \theta, t),
\end{aligned}
$$

with $\theta=\theta(\rho, \phi, t)$. Moreover, the transformed system (3.5) is also reversible with respect to $G_{1}:(\rho, \phi) \mapsto(\rho,-\phi)$.

Proof. Define the transformation $\Psi_{1}$ by

$$
\Psi_{1}: \rho=\rho, \quad \phi=\theta+H_{1}(\rho, \theta, t),
$$

with

$$
H_{1}=-\rho^{(2 m+2) \beta-1} \cdot \int_{0}^{t} g_{1}(\theta, s) d s \in \mathcal{F}((2 m+2) \beta-1) .
$$

Then, under this transformation, the system (3.4) is transformed into

$$
\begin{aligned}
& \dot{\rho}=c_{1} \rho^{(2 m+2) \beta} S^{2 n+1}\left(\phi T_{0}\right) C^{2 m+1}\left(\phi T_{0}\right)+\rho^{(2 m+2) \beta} \xi_{1}(\phi, t)+\xi_{2}(\rho, \phi, t), \\
& \dot{\theta}=c_{2} \rho^{(2 m+2) \beta-1} g(\phi)+\eta_{1}(\rho, \phi, t),
\end{aligned}
$$

where

$$
\begin{aligned}
\xi_{1}(\phi, t)= & h_{1}(\phi, t), \\
\xi_{2}(\rho, \phi, t)= & c_{1} \rho^{(2 m+2) \beta}\left[S^{2 n+1}\left(\theta T_{0}\right) C^{2 m+1}\left(\theta T_{0}\right)-S^{2 n+1}\left(\phi T_{0}\right) C^{2 m+1}\left(\phi T_{0}\right)\right] \\
& \quad+\rho^{(2 m+2) \beta}\left[h_{1}(\theta, t)-h_{1}(\phi, t)\right]+h_{2}(\rho, \theta, t), \\
\eta_{1}(\rho, \phi, t)= & c_{2} \rho^{(2 m+2) \beta-1}[g(\theta)-g(\phi)]+g_{2}(\rho, \theta, t) .
\end{aligned}
$$

From (3.4) and (3.8), it follows that

$$
H_{1}(\rho,-\theta,-t)=-H_{1}(\rho, \theta, t) .
$$

If $\rho$ is sufficiently small, the transformation $\Psi_{1}$ is invertible and the system (3.9) is reversible with respect to $G_{1}:(\rho, \phi) \mapsto(\rho,-\phi)$.

Lemma 3.2. There is a transformation $\Psi_{2}:(\rho, \phi) \mapsto(\mu, \phi)$ such that the transformed system of (3.5) is of the form

$$
\begin{aligned}
& \dot{\mu}=\Gamma_{1}(\mu, \phi, t)=c_{1} \mu^{(2 m+2) \beta} S^{2 n+1}\left(\phi T_{0}\right) C^{2 m+1}\left(\phi T_{0}\right)+\zeta(\mu, \phi, t), \\
& \dot{\phi}=\Gamma_{2}(\mu, \phi, t)=c_{2} \mu^{(2 m+2) \beta-1} g(\phi)+\sigma(\mu, \phi, t),
\end{aligned}
$$

where

$$
\begin{aligned}
& \zeta(\mu, \phi, t)=c_{1} S^{2 n+1}\left(\phi T_{0}\right) C^{2 m+1}\left(\phi T_{0}\right) \cdot\left(\rho^{(2 m+2) \beta}-\mu^{(2 m+2) \beta}\right)+\xi_{2}(\rho, \phi, t), \\
& \sigma(\mu, \phi, t)=c_{2} g(\phi) \cdot\left(\rho^{(2 m+2) \beta-1}-\mu^{(2 m+2) \beta-1}\right)+\eta_{1}(\rho, \phi, t),
\end{aligned}
$$

with $\rho=\rho(\mu, \phi, t)$. Moreover, the system (3.10) is reversible with respect to $G_{2}:(\mu, \phi) \mapsto(\mu,-\phi)$. 
Proof. Similar to the proof of Lemma 3.1, we define the transformation $\Psi_{2}$ by

$$
\Psi_{2}: \mu=\rho+H_{2}(\rho, \phi, t), \quad \phi=\phi,
$$

with

$$
H_{2}=-\rho^{(2 m+2) \beta} \cdot \int_{0}^{t} \xi_{1}(\phi, s) d s \in \mathcal{F}((2 m+2) \beta) .
$$

Then, under this transformation, the system (3.5) is transformed into

$$
\begin{aligned}
& \dot{\mu}=c_{1} \mu^{(2 m+2) \beta} S^{2 n+1}\left(\phi T_{0}\right) C^{2 m+1}\left(\phi T_{0}\right)+\zeta(\mu, \phi, t), \\
& \dot{\phi}=c_{2} \mu^{(2 m+2) \beta-1} g(\phi)+\sigma(\mu, \phi, t),
\end{aligned}
$$

where

$$
\begin{aligned}
\zeta(\mu, \phi, t)=c_{1}\left[\rho^{(2 m+2) \beta}-\mu^{(2 m+2) \beta}\right] S^{2 n-1}\left(\phi T_{0}\right) C^{2 m+1}\left(\phi T_{0}\right) \\
+\xi_{2}(\rho, \phi, t)-\xi_{2}(\mu, \phi, t), \\
\sigma(\mu, \phi, t)=c_{2}\left[\rho^{(2 m+2) \beta-1}-\mu^{(2 m+2) \beta-1}\right] g(\phi)+\eta_{1}(\rho, \theta, t)-\eta(\mu, \phi, t) .
\end{aligned}
$$

with $\rho=\rho(\mu, \phi, t)$ by (3.12). From (3.5) and (3.8), it follows that

$$
H_{2}(\rho,-\phi,-t)=H_{2}(\rho, \phi, t) .
$$

If $\rho$ is sufficiently small, the transformation $\Psi_{2}$ is invertible and the system (3.14) is reversible with respect to $G_{2}:(\mu, \phi) \mapsto(\mu,-\phi)$.

If $\mu$ is sufficiently small, from (3.11) it follows that

$$
\begin{array}{r}
\zeta(\mu, \phi, t)=o\left(\mu^{(2 m+2) \beta}\right), \\
\sigma(\mu, \phi, t)=o\left(\mu^{(2 m+2) \beta-1}\right) .
\end{array}
$$

From the equality $(m+1) S^{2 n+2}(t)+(n+1) C^{2 m+2}(t)=n+1, \forall t \in \mathbf{R}$, it follows that $0 \leq S^{2 n+2}\left(\phi T_{0}\right) \leq \frac{n+1}{m+1}$. Hence, the function $g(\phi)$ is real analytic, 1-periodic and changes the sign. In the consequence there exist $\phi^{*} \in[0,1]$ and $0<v<1$ such that $g\left(\phi^{*}\right)=0$ and

$$
g^{\prime}(\phi)=-(2 n+2) T_{0} \cdot S^{2 n+1}\left(\phi T_{0}\right) C^{2 m+1}\left(\phi T_{0}\right)<0
$$

if $\left|\phi-\phi^{*}\right| \leq v$. In fact, from the equation (2.1), we have

$$
\begin{aligned}
& S(0)=S\left(T_{0} / 2\right)=0, \quad S\left(T_{0} / 4\right)=-S\left(3 T_{0} / 4\right)=\left(\frac{n+1}{m+1}\right)^{\frac{1}{2 n+2}}, \\
& C(0)=-C\left(T_{0} / 2\right)=1, \quad C\left(T_{0} / 4\right)=C\left(3 T_{0} / 4\right)=0,
\end{aligned}
$$

and

$$
\begin{aligned}
& S(t)>0, \quad 0<t<T_{0} / 2 \quad \text { and } \quad S(t)<0, \quad T_{0} / 2<t<T_{0}, \\
& C(t)>0, \quad-T_{0} / 4<t<T_{0} / 4 \quad \text { and } \quad C(t)<0, \quad T_{0} / 4<t<3 T_{0} / 4 .
\end{aligned}
$$

Hence, we can choose that $\phi^{*} \in\left(0, \frac{1}{4}\right)$ and $v>0$ such that $g\left(\phi^{*}\right)=0$ and $\left\{\phi:\left|\phi-\phi^{*}\right| \leq v\right\} \subset\left(0, \frac{1}{4}\right)$. From the definitions of $v$ and $\phi^{*}$, it follows that $g(\phi)>0, \forall \phi \in\left(\phi^{*}-v, \phi^{*}\right)$ and $g(\phi)<0, \forall \phi \in\left(\phi^{*}, \phi^{*}+v\right)$.

Let

$$
\mathcal{K}_{\delta_{0}, v}=\left\{(\mu, \phi) \in \mathbf{R}^{+} \times \mathbf{S}^{1}: \mu<\delta_{0},\left|\phi-\phi^{*}\right|<v\right\} .
$$

Then, if $\delta_{0}$ is sufficiently small, on the set $\mathcal{K}_{\delta_{0}, v}$, we have

$$
\Gamma_{1}(\mu, \phi, t) \geq \frac{1}{2} c_{1} \mu^{(2 m+2) \beta}>0,
$$


and

$$
\begin{aligned}
& \Gamma_{2}(\mu, \phi, t)>0, \quad \text { for } 0<\mu \leq \delta_{0}, \quad-v \leq \phi-\phi^{*} \leq-\frac{1}{2} v, \\
& \Gamma_{2}(\mu, \phi, t)<0, \quad \text { for } 0<\mu \leq \delta_{0}, \quad \frac{1}{2} v \leq \phi-\phi^{*} \leq v .
\end{aligned}
$$

Lemma 3.3. Suppose $\left(\mu\left(t, \mu_{0}, \phi_{0}\right), \phi\left(t, \mu_{0}, \phi_{0}\right)\right)$ is the solution of (3.10) with the initial condition: $\left(\mu\left(0, \mu_{0}, \phi_{0}\right), \phi\left(0, \mu_{0}, \phi_{0}\right)\right)=\left(\mu_{0}, \phi_{0}\right) \in \mathcal{K}_{\delta_{0}, v}$. Then there is $T>0$ such that

$$
\left(\mu\left(T, \mu_{0}, \phi_{0}\right), \phi\left(T, \mu_{0}, \phi_{0}\right)\right) \in \partial \mathcal{K}_{\delta_{0}, v}
$$

Proof. By contradiction, we assume

$$
\left(\mu\left(t, \mu_{0}, \phi_{0}\right), \phi\left(t, \mu_{0}, \phi_{0}\right)\right) \in \mathcal{K}_{\delta_{0}, v} \quad \text { for all } t \geq 0 .
$$

From (3.18) we have $\mu\left(t, \mu_{0}, \phi_{0}\right) \geq \mu_{0}$ for $t>0$ and

$$
\begin{aligned}
\delta_{0} & \geq \mu\left(t, \mu_{0}, \phi_{0}\right)=\mu_{0}+\int_{0}^{t} \Gamma_{1}\left(\mu\left(s, \mu_{0}, \phi_{0}\right), \phi\left(s, \mu_{0}, \phi_{0}\right), s\right) d s \\
& \geq \mu_{0}+t \cdot \frac{1}{2} c_{1} \mu_{0}^{(2 m+2) \beta} \rightarrow+\infty,
\end{aligned}
$$

as $t \rightarrow+\infty$. We contradict (3.20).

Lemma 3.4. There is $t_{1}>0$ such that

$$
\mu\left(t_{1}, \mu_{0}, \phi_{0}\right)=\delta_{0}
$$

provided $\left(\mu_{0}, \phi_{0}\right) \in \mathcal{K}_{\delta_{0}, \frac{1}{2} v}$.

Proof. From Lemma 3.3, there is a constant $\mathcal{T}\left(\mu_{0}, \phi_{0}\right)>0$ such that

$$
\left(\mu\left(t, \mu_{0}, \phi_{0}\right), \phi\left(t, \mu_{0}, \phi_{0}\right)\right) \in \mathcal{K}_{\delta_{0}, v} \quad \text { for all } 0 \leq t<\mathcal{T},
$$

and

$$
\left(\mu\left(\mathcal{T}, \mu_{0}, \phi_{0}\right), \phi\left(\mathcal{T}, \mu_{0}, \phi_{0}\right)\right) \in \partial \mathcal{K}_{\delta_{0}, v}
$$

Now, we show that

$$
\mu\left(\mathcal{T}, \mu_{0}, \phi_{0}\right)=\delta_{0} .
$$

In fact, if not, from (3.21) and (3.22), it follows that

$$
\left|\phi\left(\mathcal{T}, \mu_{0}, \phi_{0}\right)-\phi^{*}\right|=v,
$$

and

$$
\mu\left(t, \mu_{0}, \phi_{0}\right)<\delta_{0}, \quad \text { for all } 0 \leq t \leq \mathcal{T} .
$$

Without loss of generality, we assume

$$
\phi\left(\mathcal{T}, \mu_{0}, \phi_{0}\right)=\phi^{*}+v .
$$

Then, there is $\tau, 0<\tau<\mathcal{T}$ such that

$$
\phi\left(\tau, \mu_{0}, \phi_{0}\right)=\phi^{*}+\frac{1}{2} v,
$$

and

$$
\phi^{*}+\frac{1}{2} v \leq \phi\left(t, \mu_{0}, \phi_{0}\right)<\phi^{*}+v, \quad \text { for all } \tau \leq t \leq \mathcal{T} .
$$

Hence, $\left.\frac{d}{d t} \phi\left(t, \mu_{0}, \phi_{0}\right)\right|_{t=\tau} \geq 0$. But, from (3.21), (3.22), (3.19) and the equation (3.10), we have

$$
\left.\frac{d}{d t} \phi\left(t, \mu_{0}, \phi_{0}\right)\right|_{t=\tau}=\Gamma_{2}\left(\mu\left(\tau, \mu_{0}, \phi_{0}\right), \phi^{*}+\frac{1}{2} v, \tau\right)<0,
$$

which yields a contradiction. 
Now, we shall prove the statement of Proposition 3.1.

Proof of Proposition 3.1. From the above discussions, we obtain the transformation $\Sigma:(\mu, \phi) \mapsto(x, y)$ given by

$$
x=\zeta_{1}(\mu, \phi, t), \quad y=\zeta_{2}(\mu, \phi, t) .
$$

Moreover, $0<x^{2}+y^{2} \ll 1 \Leftrightarrow 0<\mu \ll 1$. Under this transformation $\Sigma$, the system (1.6) is transformed into (3.10).

We assume the equilibrium $(0,0)$ of $(1.6)$ is stable in the Liapunov sense. That is, for every neighbourhood $U$ of the origin, one can find a smaller neighbourhood $V$ of the origin, $V \subset U$, such that

$$
\left(x\left(t, x_{0}, y_{0}\right), y\left(t, x_{0}, y_{0}\right)\right) \in U \quad \text { for all } t \in \mathbf{R},
$$

provided the initial point $\left(x_{0}, y_{0}\right) \in V$. This statement is equivalent to the following one:

For every $\varepsilon>0$, one can find a constant $\delta>0$ such that, if $0<\mu_{0}<\delta \leq \varepsilon$,

$$
\mu\left(t, \mu_{0}, \phi_{0}\right)<\varepsilon \text { for all } t \in \mathbf{R},
$$

where $\left(\mu\left(t, \mu_{0}, \phi_{0}\right), \phi\left(t, \mu_{0}, \phi_{0}\right)\right)$ is the solution of (3.10) with the initial condition: $\left(\mu\left(0, \mu_{0}, \phi_{0}\right), \phi\left(0, \mu_{0}, \phi_{0}\right)\right)=\left(\mu_{0}, \phi_{0}\right)$.

Now, we choose $\varepsilon=\frac{1}{2} \delta_{0}>0, \delta_{0}$ is given in the definition of $\mathcal{K}_{\delta_{0}, v}$. From Lemma 3.4, it follows that if $\left(\mu_{0}, \phi_{0}\right) \in \mathcal{K}_{\delta_{0}, \frac{1}{2} v}$ and $0<\mu_{0} \leq \delta$, we can find a constant $\tau>0$ such that

$$
\mu\left(\tau, \mu_{0}, \phi_{0}\right)=\delta_{0}>\varepsilon
$$

which yields a contradiction. This completes the proof of Proposition 3.1.

Combining Propositions 2.1 and 3.1, we obtain the statement of the theorem in the introduction.

Remarks. 1. Using the method of this paper, we can study the stability of the trivial solution $(x, y)=(0,0)$ of the following Hamiltonian system

$$
\dot{x}=\frac{\partial H}{\partial y}, \quad \dot{y}=-\frac{\partial H}{\partial x},
$$

with the Hamiltonian $H=a(t) y^{2 m+2}+b(t) x^{2 n+2}+h(x, y, t)$. If $h$ has the property $\mathcal{P}(m, n)$ and $\int_{0}^{1} a(t) d t \cdot \int_{0}^{1} b(t) d t \neq 0$, then the trivial solutions $(x, y)=(0,0)$ is stable in the Liapunov sense if and only if

$$
\int_{0}^{1} a(t) d t \cdot \int_{0}^{1} b(t) d t>0 .
$$

2. In this paper, we assume that $m+n \geq 1$ in (1.6). If $m=n=0$, it seems that the method in the paper cannot be used to study the stability of the equilibrium of (1.6). In this case, the stability of the equilibrium depends not only on the properties of $a(t), b(t)$, but the properties of $f_{1}, f_{2}$. We shall study it in a separate paper.

3. The statements of our theorem are also valid for the following reversible system:

$$
\begin{aligned}
& \dot{x}=w_{1}(t) x^{2 n+1}+a(t) y^{2 m+1}+f_{1}(x, y, t), \\
& \dot{y}=-b(t) x^{2 n+1}-w_{2}(t) y^{2 m+1}+f_{2}(x, y, t),
\end{aligned}
$$


where $m, n \in \mathbf{Z}_{+}, m+n \geq 1, a(t)$ and $b(t)$ are continuous, even and 1-periodic functions in the time variable $t ; w_{1}(t)$ and $w_{2}(t)$ are continuous, odd and 1-periodic $t ; f_{1}$ and $f_{2}$ are real analytic in a neighbourhood of the origin $(0,0)$ of $(x, y)$-plane and continuous 1-periodic in $t$. We also assume that the above system is reversible with respect to the involution $G:(x, y) \mapsto(-x, y)$. More precisely, we have

Theorem 2. Assume that $\int_{0}^{1} a(t) d t \cdot \int_{0}^{1} b(t) d t \neq 0$ and $f_{1}, f_{2}$ satisfy the property $\mathcal{P}(m, n)$. Then the following statements are equivalent:

(1) The trivial solution $(x, y)=(0,0)$ of the equation (3.24) is stable;

(2) $\int_{0}^{1} a(t) d t \cdot \int_{0}^{1} b(t) d t>0$.

Moreover, if $\int_{0}^{1} a(t) d t \cdot \int_{0}^{1} b(t) d t>0$, there is at least two subharmonic solutions of (3.24) with minimal period $k$ for every sufficiently large $k \in \mathbf{N}$ in a neighborhood of the equilibrium of (3.24).

The proof of this theorem is similar to the proof of Theorem 1.

\section{ACKNOWLEDGEMENTS}

I would like to acknowledge the help of Professor Tongren Ding in clarifying my proofs. I also want to thank Dr. Mingliang Pei, who drew my attention to the work of R. Ortega.

\section{APPENDIX}

In this appendix, we give some definitions and a lemma which have been used in this paper.

Definition A.1. Let $f: \mathbf{R}^{n} \times \mathbf{R} \rightarrow \mathbf{R}^{n}$ be periodic in the last variable. The system

$$
\dot{x}=f(x, t)
$$

is called reversible if there is a mapping $G: \mathbf{R}^{n} \rightarrow \mathbf{R}^{n}$ such that

$$
G^{2}=\mathrm{id}, \quad D G \circ f(G x,-t)=-f(x, t) .
$$

Definition A.2. Assume that $M$ is an invertible mapping of $\mathbf{R}^{n}$ and $G$ is defined in Definition A.1. The mapping $M$ is called reversible with respect to $G$ if the equality

$$
G \circ M \circ G=M^{-1}
$$

holds.

From the above definitions, it follows that the Poincare mapping of some reversible system is also reversible with respect to the same involution.

Now, we introduce the concept of $G$-invariant for the transformations of $\mathbf{R}^{n}$ which depend on time $t$.

Definition A.3. Assume that $T(x, t)$ is an invertible transformation of $\mathbf{R}^{n}$ for any fixed $t$ and $G$ is an involution of $\mathbf{R}^{n} . T(x, t)$ is called $G$-invariant if the equality

$$
G \circ T(x, t)=T(G(x),-t)
$$

holds. 
Denote $T^{-1}(x, t)$ by the inverse of $T$, i.e.,

$$
T^{-1}(T(x, t), t)=T\left(T^{-1}(x, t), t\right)=x .
$$

If $T$ is $G$-invariant, then so is $T^{-1}(x, t)$.

From the definition, we have

Lemma A.1. Consider a system in $\mathbf{R}^{n} \times \mathbf{R}$ is of the form

$$
\dot{x}=f(x, t),
$$

which is a reversible system with respect to $G$. Suppose $T(x, t): \mathbf{R}^{n} \rightarrow \mathbf{R}^{n}$ is $G$ invariant, then the transformed system of (A.2) under the transformation $T$ is also reversible with respect to $G$.

Proof. It is easy to see that the transformed system is of the form

$$
\dot{y}=F(y, t),
$$

where

$$
F(y, t)=\frac{\partial T}{\partial x}\left(T^{-1}(y, t), t\right) \cdot f\left(T^{-1}(y, t), t\right)+\frac{\partial T}{\partial t}\left(T^{-1}(y, t), t\right) .
$$

From the equality (A.1), it follows that

$$
D G(T(x, t)) \cdot \frac{\partial T}{\partial x}(x, t)=\frac{\partial T}{\partial x}(G(x),-t) \cdot D G(x)
$$

and

$$
D G(T(x, t)) \cdot \frac{\partial T}{\partial t}(x, t)=-\frac{\partial T}{\partial t}(G(x),-t)
$$

Hence,

$$
\begin{aligned}
D G(y) \cdot F(y, t)= & D G(y) \cdot \frac{\partial T}{\partial x}\left(T^{-1}(y, t), t\right) \cdot f\left(T^{-1}(y, t), t\right) \\
& +D G(y) \cdot \frac{\partial T}{\partial t}\left(T^{-1}(y, t), t\right) \\
= & \frac{\partial T}{\partial x}\left(G\left(T^{-1}(y, t)\right),-t\right) \cdot D G\left(T^{-1}(y, t)\right) \cdot f\left(T^{-1}(y, t), t\right) \\
& -\frac{\partial T}{\partial t}\left(G\left(T^{-1}(y, t)\right),-t\right) .
\end{aligned}
$$

Because the system (A.2) is reversible and $T^{-1}$ is $G$-invariant, we have

$$
\begin{aligned}
D G\left(T^{-1}(y, t)\right) \cdot f\left(T^{-1}(y, t), t\right) & =-f\left(G\left(T^{-1}(y, t)\right),-t\right) \\
& =-f\left(T^{-1}(G(y),-t),-t\right),
\end{aligned}
$$

which yields that

$$
D G(y) \cdot F(y, t)=-F(G(y),-t) .
$$

This means that the transformed system (A.2) is reversible with respect to $G$. 


\section{REFERENCES}

[1] L. Cesari, Asymptotic Behavior and Stability Problems in Ordinary Differential Equations, Springer-Verlag, New York, Berlin, 1971. MR 50:2582

[2] R. Dieckerhoff and E. Zehnder, Boundedness of solutions via the twist theorem, Ann. Scula. Norm. Sup. Pisa Cl. Sci. (4) 14 (1987), 79-95. MR 89e:34066

[3] B. Liu, The stability of the equilibrium of a conservative system, J. Math. Anal. Appl. 202 (1996), 133-149. MR 97e:34094

[4] W. Magnus and S. Winkler, Hill's Equation, Dover, New York, 1979. MR 80k:34001

[5] J. Moser, On invariant curves of area-preserving mappings of annulus, Nachr. Akad. Wiss. Göttingen Math.-Phys. Kl. 1962, 1-20. MR 26:5255

[6] Stable and Random Motions in Dynamical Systems, Princeton Univ. Press, Princeton, N.J., 1973. MR 56:1355

[7] R. Ortega, The stability of the equilibrium of a nonlinear Hill's equation, SIAM J. Math. Anal. 25 (1994), 1393-1401. MR 95g:34071

[8] M. Sevryuk, Reversible Systems, Springer-Verlag, New York, Berlin, 1986. MR 88b:58058

[9] L. Siegel and J. Moser, Lectures on Celestial Mechanics, Springer-Verlag, New York, Berlin, 1971. MR 58:19464

Department of Mathematics, Peking University, Beijing 100871, China

E-mail address: bliu@pku.edu.cn 